\title{
The comparative study with lixisenatide /glargine combination therapy versus twice-daily premixed insulin therapy/DPP-4 inhibitor on glycemic variability, and feeding behavior in obese patients with type 2 diabetes mellitus
}

\author{
Fumihiro Ochi ${ }^{1}$, Tomoyuki Katsuno ${ }^{2 *}$, Masaru Tokuda ${ }^{1}$, Yoshiki Kusunoki ${ }^{1}$, Mitsuyoshi Namba ${ }^{1}$ and Hidenori Koyama ${ }^{1}$ \\ ${ }^{1}$ Division of Diabetes, Endocrinology and Metabolism, Department of Internal Medicine, Hyogo College of Medicine, Nishinomiya 663-8501, Japan \\ ${ }^{2}$ Department of Occupational Therapy, School of Rehabilitation, Hyogo University of Health Sciences, Kobe 650-8530, Japan
}

\begin{abstract}
Ten obese patients with type 2 diabetes being treated with twice-daily injections of premixed insulin/DPP-4 inhibitor were switched to the combined glargine/ lixisenatide $(20 \mu \mathrm{g} / \mathrm{day})(\mathrm{G}+\mathrm{L})$ therapy; the efficacy and safety of the combination regime were evaluated. Blood glucose fluctuations were evaluated with continuous glucose monitoring (CGM) before and after switching the treatment. Treatment-related quality of life (QOL) was assessed using the insulin therapy-related QOL (ITR-QOL) and diabetes therapy-related QOL (DTR-QOL) questionnaires, and eating behavior using a dietary behavior questionnaire. Sixteen weeks after switching to $\mathrm{G}+\mathrm{L}$ therapy, fasting blood glucose levels improved significantly from 179 (155-197) to $132(127-151) \mathrm{mg} / \mathrm{dL}$ ( $\mathrm{p}<0.01)$, as did glycoalbumin levels, from $20.2 \%(18.7 \%-22.0 \%)$ to $19.7 \%(18.4 \%-21.3 \%)$. Fasting C-peptide immunoreactivity decreased significantly from $1.66(1.35-2.18)$ to $1.40(0.95-1.73) \mathrm{ng} / \mathrm{ml}$ $(\mathrm{p}<0.01)$, as did body weight, from $68.0(62.9-70.8)$ to $66.1(59.8-69.8) \mathrm{kg}$. CGM results indicated that the area under the curve in $0-3 \mathrm{~h}$ post-breakfast improved significantly from $549.6(498.7-588.3)$ to $379.0(268.0-436.0) \mathrm{h} \cdot \mathrm{mg} / \mathrm{dL}(\mathrm{p}=0.005)$ and $\mathrm{M}$ values also improved significantly from 86.1 (41.0-104.5) to 37.2 (16.1-73.0). Significant improvements were noted in certain sections of ITR-QOL (general, social activity, and emotions), DTR-QOL (anxiety related to treatment and level of satisfaction with treatment), and the dietary behavior questionnaire, (total score, food motivation, eating as a diversion, and diet regularity). Changing from a twice-daily insulin regimen to $\mathrm{G}+\mathrm{L}$ therapy in obese patients with type 2 diabetes improved blood glucose management, decreased body weight, and improved QOL, including patient adherence and dietary behavior.
\end{abstract}

\section{Introduction}

A twice-daily injection regimen of premixed insulin has been often employed rather than frequent insulin injection therapy (frequent injection method) due to its simplicity. However, lack of flexibility in changing the method of treatment when blood glucose cannot be sufficiently managed has been reported by American Diabetes Association and the European Association for the Study of Diabetes [1]. In type 2 diabetes treatment in Japan, the mixed twice-daily method has certainly contributed to improving patient adherence compared to frequent injection method. However, mixed insulin needs to be actually mixed before injections, and, depending on the patient, the trouble associated with adjusting the amount could lead to hypoglycemic tendencies, intensified sense of hunger, and excessive food ingestion leading to increased body weight gain. These factors then make longterm management difficult, particularly obese patients.

Obesity that develops along with insulin treatment often causes specific changes to dietary behavior in the form of deviation and/ or habits. Deviation refers to deviation in perceptions, the sensation of fullness, and amount of food ingested. Habits refer to bad dietary behavior habits. These then have a negative impact on the execution of dietary and exercise therapies and their effects and significantly impede treatment for obesity and diabetes.
Glucagon-like peptide-1 receptor agonists (GLP-1 RA) promote the glucose-dependent secretion of insulin and also inhibit excessive glucagon secretion, thereby improving blood glucose and producing appetite-suppressing tendencies by acting on the central nervous and digestive systems. Associated body weight loss might contribute to the long-term management of diabetes treatment. We have previously reported that in overweight patients with type 2 diabetes, GLP-1 RA improves dietary behavior, leading to weight loss and blood glucose improvement [2]. The GLP-1 RA lixisenatide has a structure similar to exendin-4. By deleting the 38th position Pro and adding 6 Lys to the C-terminal, resistance to DPP-4 has been intensified, and stability in blood has also been improved when compared to exendin-4. Its halflife in blood is approximately $2.45 \mathrm{~h}$, and it is classified as a short-acting GLP-1 RA based on differences in reaction time [3]. The Get Goal-L

${ }^{\star}$ Correspondence to: Tomoyuki Katsuno, Department of Occupational Therapy, School of Rehabilitation, Hyogo University of Health Sciences, Kobe 650-8530, Japan, Tel: +81 78304 3063; Email: katsunoa@hyo-med.ac.jp

Key words: premixed insulin, basal insulin, Short-acting GLP-1 receptor agonists

Received: August 28, 2018; Accepted: September 04, 2018; Published: September 07, 2018 
Ochi F (2018) The comparative study with lixisenatide /glargine combination therapy versus twice-daily premixed insulin therapy/DPP-4 inhibitor on glycemic variability, and feeding behavior in obese patients with type 2 diabetes mellitus

Asia study that targeted 311 Asian patients with type 2 diabetes being treated with lixisenatide found that it exhibited strong improvement of postprandial glucose levels and led to significant improvement in weight loss and HbA1c levels [4]. GLP-1 receptor agonists and DPP-4 inhibitors lead to greater improvements in blood glucose management for patients who have improved fasting blood glucose levels at the time of starting administration [5].

Sustained-release type insulin has been available as a basal insulin preparation that improves fasting blood glucose levels by means of long-acting effects over $24 \mathrm{~h}$. In Japan, this type of insulin has been approved for combined use with lixisenatide.

Accordingly, although the combined use of sustained-release insulin with lixisenatide is anticipated to improve both of fasting and postprandial blood glucose levels, few reports in Japan have investigated in detail the effects of switching from a twice-daily injection regimen of mixed-type insulin to combined therapy with lixisenatide and sustained-release type insulin. We switched overweight patients with type 2 diabetes who exhibited insufficient blood glucose control with the mixed twice-daily method to a regimen of one dose in the morning of lixisenatide and glargine, and then compared the efficacy and safety of both methods based on changes in blood glucose control, body weight, blood pressure, and lipid profiles. QOL and changes in dietary behavior were also assessed using questionnaires. We also report the results for the meal tolerance test (MTT) with test meals and blood glucose fluctuations according to continuous glucose monitoring (CGM) performed before and after changing the method of treatment.

\section{Materials and Methods}

\section{Subjects}

We enrolled overweight patients with type 2 diabetes who were attending outpatient examinations between August 2014 and June 2017 at the Division of Diabetes, Endocrinology and Metabolism in the Hyogo College of Medicine. These patients underwent diet and exercise therapy in addition to pharmacotherapy (twice-daily injections of premixed insulin with DPP-4 inhibitor and/or using of other oral hypoglycemic agents) for at least 12 weeks but had HbAlc levels $>7.0 \%$. The inclusion criteria were as follows: 1) patients taking twice-daily injections of premixed insulin with DPP-4 inhibitor; 2) HbAlc $>7.0 \%$ (NGSP); 3) 20-80 years old; and 4) body mass index (BMI) $>25$. The exclusion criteria were as follows: 1) type 1 diabetes; 2 ) history of severe ketosis, diabetic coma or pre-coma in the past 6 months; 3 ) women who are or could be pregnant and women who are breastfeeding; 4) patients after a severe infection, before or after surgery, or with severe injury; 5) patients receiving whole-body administration of adrenocortical steroids; 6) patients with severely impaired liver function; 7) patients suspected to have decreased insulin secretion ability; 8) patients with pancreatitis or a history of pancreatitis; 9) patients with a malignant tumor or history of malignant tumor; and 10) patients deemed unfit by their primary physician to participate in the study for any other reason. After applying the exclusion criteria, 10 patients were included in the study (Table 1).

\section{Study Design}

The twice-daily injections of premixed insulin were switched to a once-daily pre-breakfast dose of glargine, with half of the daily total dose of premixed insulin. From then on, self-monitoring of blood glucose (SMBG) was used, and the dose of glargine was adjusted with the target of a fasting blood glucose level $<140 \mathrm{mg} / \mathrm{dL}$ for 4 weeks. After that, DPP-4 inhibitors were discontinued, and the combined
Table 1. Patient characteristics

\begin{tabular}{|l|l|}
\hline & Patient profiles $(\mathbf{n}=\mathbf{1 0})$ \\
\hline Gender (Male: Female) & $5: 5$ \\
\hline Age $(\mathrm{y})$ & $60.5(50.2-70.2)$ \\
\hline Height $(\mathrm{cm})$ & $161.5(152.4-169.5)$ \\
\hline Body weight $(\mathrm{kg})$ & $68.0(62.9-70.8)$ \\
\hline Body mass index $\left(\mathrm{kg} / \mathrm{m}^{2}\right)$ & $25.4(25.0-26.7)$ \\
\hline Duration of DM (years) & $11(7.7-19.5)$ \\
\hline HbA1c $(\mathrm{NGSP})(\%)$ & $8.2(7.6-8.5)$ \\
\hline Fasting plasma glucose $(\mathrm{mg} / \mathrm{dl})$ & $179.0(155.2-197.7)$ \\
\hline Plasma CPR (ng/ml) & $1.6(1.3-2.1)$ \\
\hline CPR index & $1.0(0.7-1.3)$ \\
\hline insulin $(75 / 25: 70 / 30: 50 / 50)$ & $3: 2: 5$ \\
\hline
\end{tabular}

use of lixisenatide with glargine was started from a once-daily dose of $10 \mu \mathrm{g}$ each morning. Each week, the dose was increased by $5 \mu \mathrm{g}$ until a maintenance dose of $20 \mu \mathrm{g}$ was reached. Lixisenatide treatment was continued for 16 weeks. After starting combined treatment with lixisenatide, the glargine dose was fixed as a rule (Figure 1).

At the start of the study (week 0 ) until the end of the study period, blood was collected every 4 weeks, and parameters including HbAlc, glycoalbumin (GA), fasting blood glucose levels, serum C-peptide immunoreactivity (CPR), and lipids (T-cholesterol, LDL, and TG) were measured. Body weight, blood pressure, and pulse rate were also measured every 4 weeks. QOL was evaluated using a questionnaire, and dietary behavior was evaluated using a dietary behavior questionnaire at weeks 0 and 16 after starting lixisenatide administration.

When evaluating the effects of treatment on daily lifestyle, the insulin therapy-related QOL (ITR-QOL) questionnaire [6] was used to evaluate insulin treatment and the diabetes therapy-related QOL (DTR-QOL) questionnaire [7] was used to evaluate QOL as a result of changing treatment. Each questionnaire was classified into five sections (ITR-QOL: general, daily lifestyle, physical symptoms, social activity, emotions; DTR-QOL: general, social activity/daily life, anxiety/dissatisfaction regarding treatment, hypoglycemia, and level of satisfaction with treatment). Scores for the individual sections and total scores were calculated, and the results for weeks 0 and 16 were compared.

Dietary behavior was evaluated with the Dietary Behavior Questionnaire [8] by the Japan Society for the Study of Obesity that is used to investigate diet-related issues. The 55 questions were classified into a total of seven sections (perception of physical constitution and weight, food motivation, eating as a diversion, sense of hunger/ fullness, way of eating, dietary content, and diet regularity). Scores for the individual sections and total scores were calculated, a diagram was created, and the results for weeks 0 and 16 were compared. The questionnaires were collected by a diabetes consultant at our hospital, and no intervention for lifestyle guidance was conducted.

In addition, the MTT using test meals was conducted in weeks 0 and 16, and blood glucose fluctuations was evaluated using Continuous Glucose Monitoring (CGM) conducted over 3 days. From $1800 \mathrm{~h}$. the day before CGM evaluation and throughout the 3-day CGM evaluation, meals were unified. The mealtimes and dietary content are described as follows. At 0800 h: JANEF ${ }^{\oplus}$ E60F18 (460 kcal, carbohydrates: 56.5 g, protein: 18.0 g, fat: 18.0 g, Kewpie Corporation, Tokyo, Japan), 1200 h: Calorie Navi 320 (NICHIREI CORPORATION, Tokyo, Japan) + $150 \mathrm{~g}$ of packed sterilized rice (total: $436 \mathrm{kcal}$, carbohydrates: $76.0 \mathrm{~g}$, protein: $24.4 \mathrm{~g}$, fat: $14.1 \mathrm{~g}$ ), $1800 \mathrm{~h}$ : Calorie Navi ${ }^{\oplus} 320+150 \mathrm{~g}$ of packed sterilized rice (total: $436 \mathrm{kcal}$, carbohydrates: $74.9 \mathrm{~g}$, protein: $22.5 \mathrm{~g}$, fat: $13.0 \mathrm{~g}$ ). 
Ochi F (2018) The comparative study with lixisenatide /glargine combination therapy versus twice-daily premixed insulin therapy/DPP-4 inhibitor on glycemic variability, and feeding behavior in obese patients with type 2 diabetes mellitus

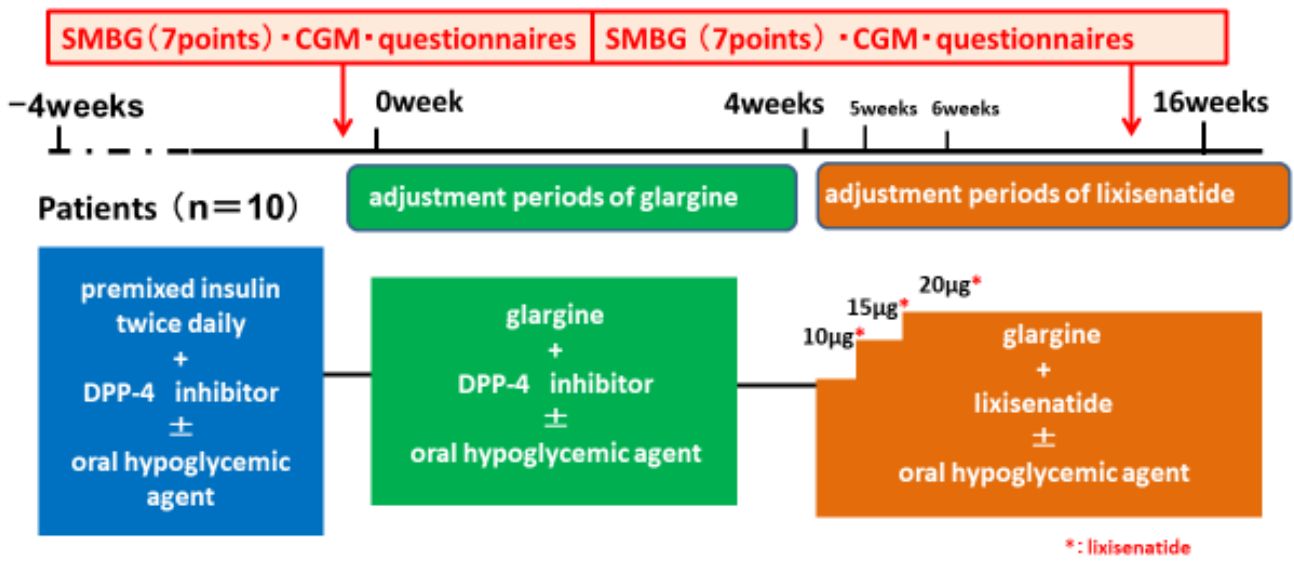

Figure 1. Study protocol

\section{Statistical Analysis}

Mini Stat-Mate (ATMS Co., Ltd., Tokyo, Japan) was used for statistical analysis in this study. The Wilcoxon signed-rank test was used to compare weeks 0 and 16 and to compare test values between the two groups, and $\mathrm{p}<0.05$ was considered to indicate a significant difference. Median values (1st-3rd quartiles) are shown.

This study was approved by the Clinical Research Ethical Review Board of the Hyogo College of Medicine (approval no.: 1836). The study was registered on the UMIN Clinical Trials Registry (UMIN Clinical Trials ID: UMIN000016628), and conducted in accordance with the Declaration of Helsinki. All subjects were given an explanation of the details of the study and voluntarily gave written informed consent to participation.

\section{Results}

Subjects consisted of 10 overweight patients with type 2 diabetes (5 males and 5 females). Mean values were $8.2 \%$ (7.6\%-8.5\%) for HbA1c, 68 (62.9-70.8) kg for body weight, and 25.4 (25-26.7) for BMI. The premixed insulin comprised a $25 / 75$ preparation for three patients (30\%), a 30/70 preparation for two patients (20\%), and a 50/50 preparation for five patients (50\%). Oral hypoglycemic agents were glimepiride for one case, metformin for nine cases, miglitol for two cases, and sitagliptin for all 10 cases (Table 1).

\section{Changes in fasting blood glucose levels, GA and HbA1c}

Comparison of weeks 0 and 16 revealed that fasting blood glucose levels improved significantly from $179(155-197) \mathrm{mg} / \mathrm{dL}$ to 132 $(127-151) \mathrm{mg} / \mathrm{dL}(\mathrm{p}<0.01)$. GA improved significantly from $20.15 \%$ $(18.70 \%-21.97 \%)$ to $19.65 \%(18.40 \%-21.32 \%)$ [p < 0.05 ]. HbAlc improved slightly from $8.20 \%(7.60 \%-8.57 \%)$ to $7.90 \%(7.25 \%-8.27 \%)$ but no significant difference was noted ( $\mathrm{p}=0.357$ [Figure 2]).

\section{Changes in fasting CPR}

Comparison of weeks 0 and 16 indicated that fasting CPR decreased significantly from $1.66(1.35-2.18) \mathrm{ng} / \mathrm{mL}$ to $1.40(0.95-1.73) \mathrm{ng} / \mathrm{mL}$ (p $<0.01)$.

\section{Changes in insulin dosage}

No significant changes were noted for total insulin dosage, which was 23 (16-29.5) units in week 0 and 24 (18.5-30.5) units at week 16.

\section{Changes in body weight}

Comparison of weeks 0 and 16 indicated that body weight decreased significantly from $68.0(62.9-70.8) \mathrm{kg}$ to $66.1(59.8-69.8) \mathrm{kg}(\mathrm{p}<0.05)$.

\section{Changes in lipids, blood pressure, and pulse rate}

Comparison of weeks 0 and 16 indicated no significant changes for lipids (total cholesterol, triglycerides, and LDL cholesterol), blood pressure, or pulse rate.

\section{Adverse events (severe hypoglycemia, etc.)}

No cases of severe hypoglycemia requiring the assistance of a third party or intravenous administration of glucose were noted during the study period. No adverse events requiring discontinuation of the study were observed.

\section{Changes in QOL according to questionnaires}

ITR-QOL (Figure 3A) questionnaire was used to assess insulin treatment-related QOL. Comparison of weeks 0 and 16 indicated that according to each of the five sections, significant improvements for the three sections of general, social activity, and emotions $(\mathrm{p}<0.05)$ were noted. Investigation of the question items composing each of the sections indicated significant improvements for the parameters of activity limitations on leisure and interests, constraints on time for starting meals, and difficulty performing injections while eating out $(\mathrm{p}<0.05$ [Figure 4A]). Although there was a tendency for decreased scores for the feeling ill question only, this may have been due to nausea experienced when patients started to take lixisenatide (Figures 3 and 4)

For the DTR-QOL questionnaire (Figure 3B), which evaluates overall treatment, significant improvements were noted for two of the five sections-anxiety regarding treatment and level of satisfaction with treatment $(\mathrm{p}<0.05)$. Investigation of the questions composing each of the sections indicated significant improvements for weight gain, hyperglycemia, blood sugar instability, decreased anxiety regarding continuing current treatment, and improved hope and level of satisfaction regarding switching the method of treatment $(\mathrm{p}<$ 0.05 [Figure 4B]). Switching treatment also brought about improved tendencies for question parameters related to hypoglycemia. In addition, trends toward significant improvements were observed in dissatisfaction with disruption of work, errands, interacting with people $(p=0.022)$, and anxiety regarding decreased range of activity $(\mathrm{p}=0.030)$. 
Ochi F (2018) The comparative study with lixisenatide/glargine combination therapy versus twice-daily premixed insulin therapy/DPP-4 inhibitor on glycemic variability, and feeding behavior in obese patients with type 2 diabetes mellitus
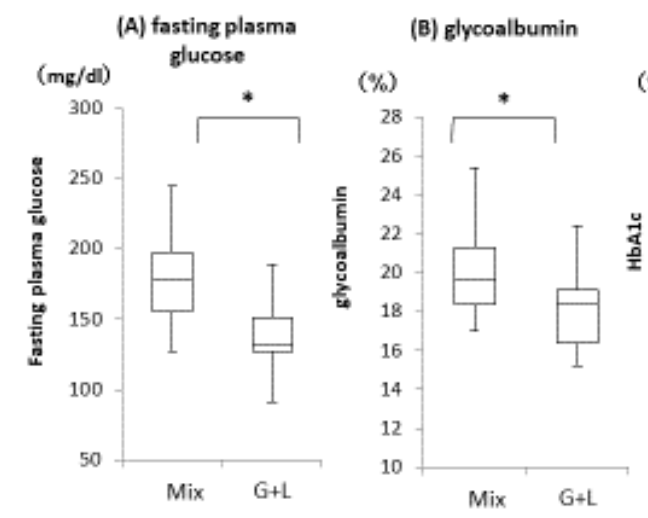

(C) Hbalc

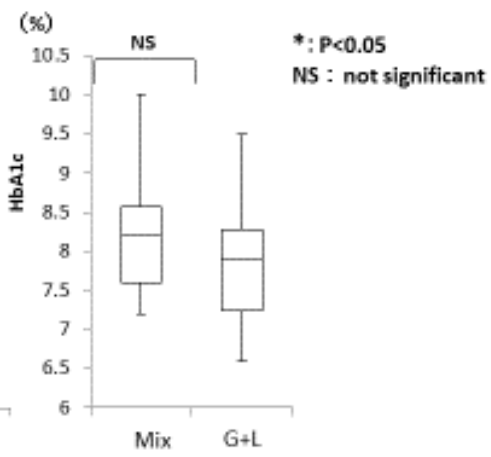

Mix: premixed insulin/DPP-4 inhibitor

$\mathrm{G}+\mathrm{L}$ : glargine / lixisenatide

Figure 2. Comparison of fasting blood glucose levels (A), glycoalbumin (B), and HbAlc (C) with twice-daily injections of premixed insulin/DPP-4 inhibitor and glargine / lixisenatide therapy

A)

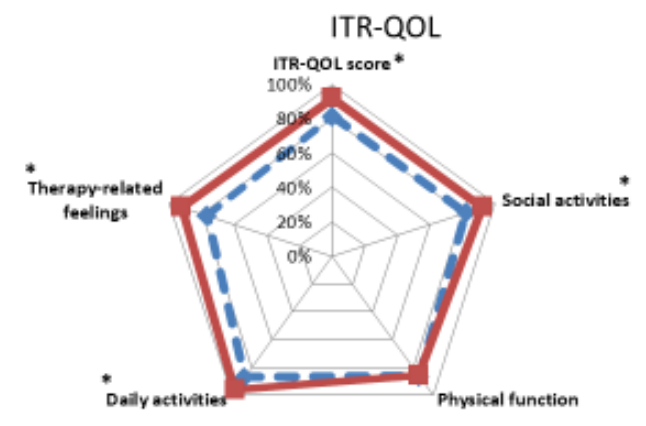

B)

DTR-QOL

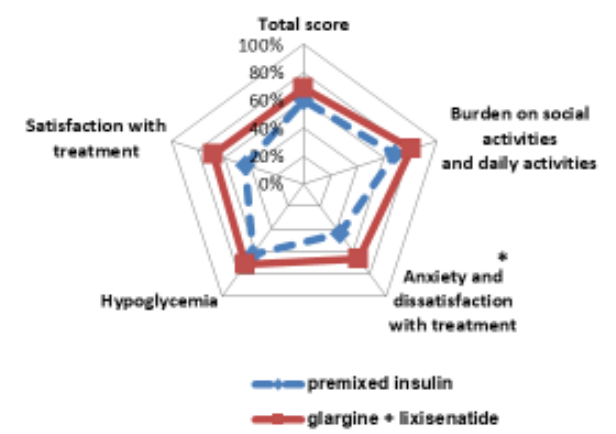

Figure 3. Comparison of twice-daily injections of premixed insulin / DPP4 inhibitor and glargine /lixisenatide therapy in each section of ITR-QOL and DTR-QOL $(* \mathrm{p}<0.05$ )

A) No. 5: Leisure activities(e.g. hobbies) are restricted because of insulin injection.

No.16: Time to start meal is restricted because of insulin injection.

No.21: It is difficult to inject insulin when I eat out.

B) Factor 2 : Anxiety and dissatisfaction with treatment

No.14: I am bothered by weight gain with my current diabetes treatment.

No.19: I have uncomfortable symptoms due to hyperglycemia.

No.21: I am dissatisfied that my blood glucose is unstable.

No.22: I am worried that complications might get worse with my current diabetes treatment.

No.23: I get anxious thinking about living while on my current diabetes treatment.

Factor 4 : Satisfaction with treatment

No.28: I am hopeful about the future with my current diabetes treatment.

No.29: With regards to diabetes treatment, 1 am satisfied with current treatment methods.

premixed insulin
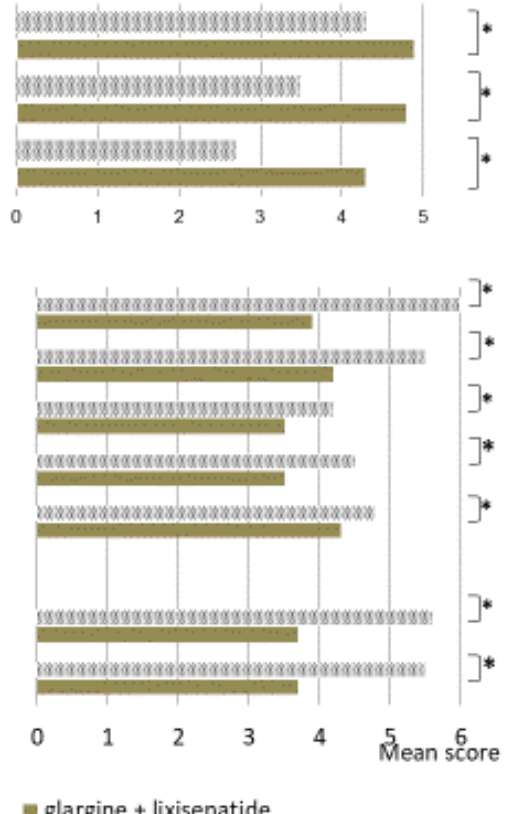

alargine + lixisenatide

Figure 4. 4A) Comparison of twice-daily injections of premixed insulin/DPP-4 inhibitor and glargine/lixisenatide therapy for questions on the ITR-QOL questionnaire (No. 5, 16, and 21), 4B) Questions on the DTR-QOL questionnaire (Factor 2: No. 14, 19, 21, 22, and 23; Factor 4: No. 28 and 29) $(* \mathrm{p}<0.05)$ 
Ochi F (2018) The comparative study with lixisenatide /glargine combination therapy versus twice-daily premixed insulin therapy/DPP-4 inhibitor on glycemic variability, and feeding behavior in obese patients with type 2 diabetes mellitus

\section{Changes in dietary behavior according to the questionnaire}

Significant improvements were observed in week 16 , compared to week 0 , in the dietary behavior questionnaire for total scores, as well as for the sections concerning food motivation, eating as a diversion, and diet regularity $(\mathrm{p}<0.05)$. Investigation of the questions comprising each of the sections indicated significant improvements for the food motivation question regarding buying extra food items, the eating as a diversion question regarding always keeping food close by, and the diet regularity question regarding dinner as the most decadent and largest meal of the day (Figure 5).

\section{Changes in blood glucose fluctuations according to CGM in MTT}

The results of measurement of changes in blood glucose levels over $24 \mathrm{~h}$ with CGM in MTT to compare weeks 0 and 16 indicated that although no significant changes were noted in mean blood glucose levels or mean amplitude of glycemic excursion (MAGE) after changing treatment, $M$ values improved significantly from $86.1(41.0-104.5)$ to $37.2(16.1-73.0[\mathrm{p}<0.05])$ as shown in Figure 6.

Furthermore, comparison of AUC at $3 \mathrm{~h}$ after each meal indicated significant improvements for post-breakfast AUC from 0-3 h ranging from $549.6(498.7-588.3) \mathrm{h} \cdot \mathrm{mg} / \mathrm{dL}$ to $379.0(268.0-436.0) \mathrm{h} \cdot \mathrm{mg} / \mathrm{dL}$ $(\mathrm{p}=0.005)$. Post-lunch and post-dinner AUC from the $0-3 \mathrm{~h}$ results showed no significant changes $(498.0[413.3-544.8] \mathrm{h} \cdot \mathrm{mg} / \mathrm{dL}$ to 453.5 [389.6-544.2] h.mg/dL, and $656.4[600.0-1032.4] \mathrm{h} \cdot \mathrm{mg} / \mathrm{dL}$ to 801.0 [671.7-959.6] h.mg/dL, respectively [Figure 7]).

\section{Discussion}

Combined therapy with basal insulin and GLP-1 RA for type 2 diabetes has been reported to offer significantly superior blood glucose control and improvement of the risks for hypoglycemia and body weight gain compared to basal-bolus therapy [9]. Since there have been few detailed investigations conducted in Japan regarding switching from the mixed twice-daily method to combined therapy with basal insulin and GLP-1RA, in obese type 2 DM we switched patients from a regimen of twice-daily injections of premixed insulin with DPP-4 inhibitor to combined therapy with a once per morning dose of glargine and lixisenatide, a short-acting GLP-1 RA and investigated the efficacy of this treatment.

In the study, changing the method of treatment from a regimen of twice-daily injections of premixed insulin with DPP-4 inhibitor improved blood glucose management, with significant improvement in fasting blood glucose levels.

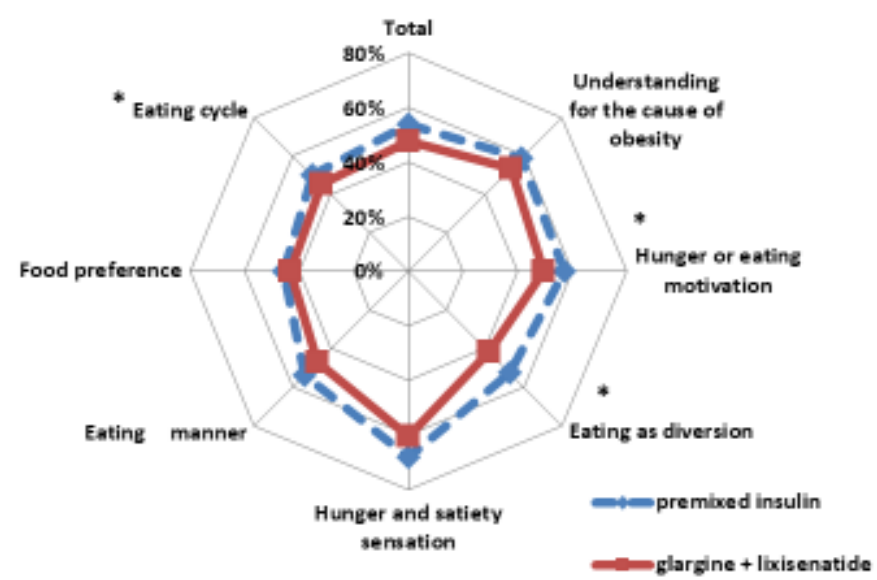

Figure 5. Comparison of twice-daily injections of premixed insulin / DPP-4 inhibitor and glargine/ lixisenatide therapy for sections on the food behavior questionnaire $(* \mathrm{p}<0.05)$

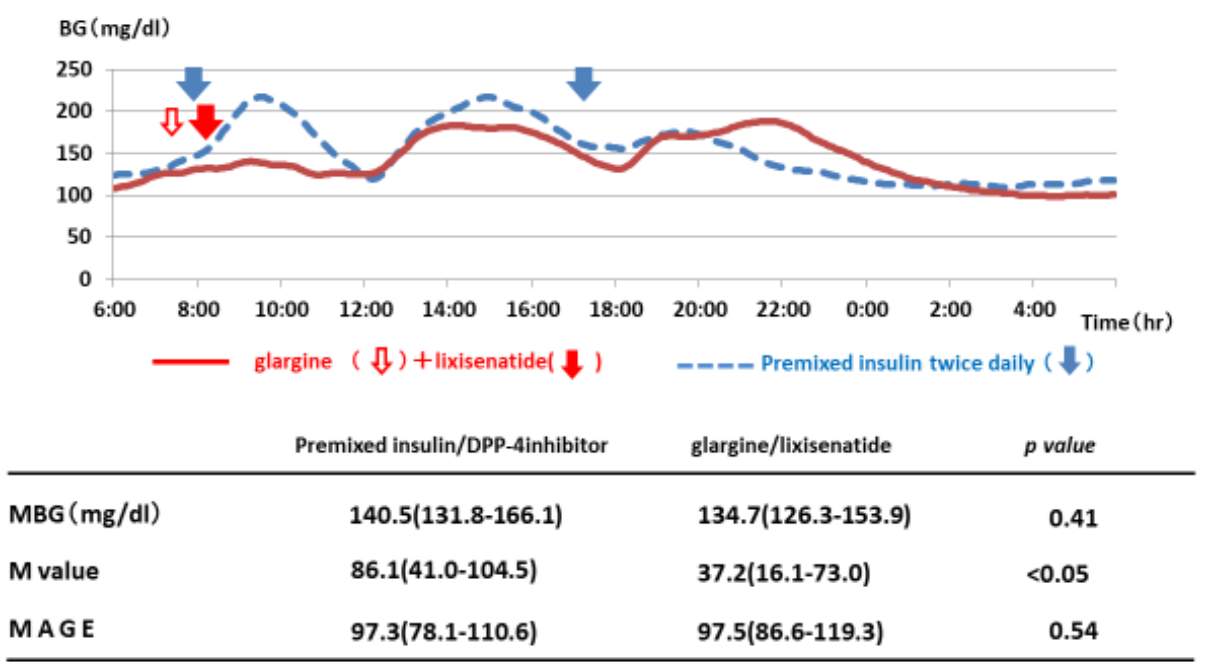

Figure 6. Comparison of twice-daily injections of premixed insulin/ DPP-4 inhibitor and glargine/lixisenatide therapy for the results of CGM combined with MMT (*p < 0.05) 
Ochi F (2018) The comparative study with lixisenatide /glargine combination therapy versus twice-daily premixed insulin therapy/DPP-4 inhibitor on glycemic variability, and feeding behavior in obese patients with type 2 diabetes mellitus
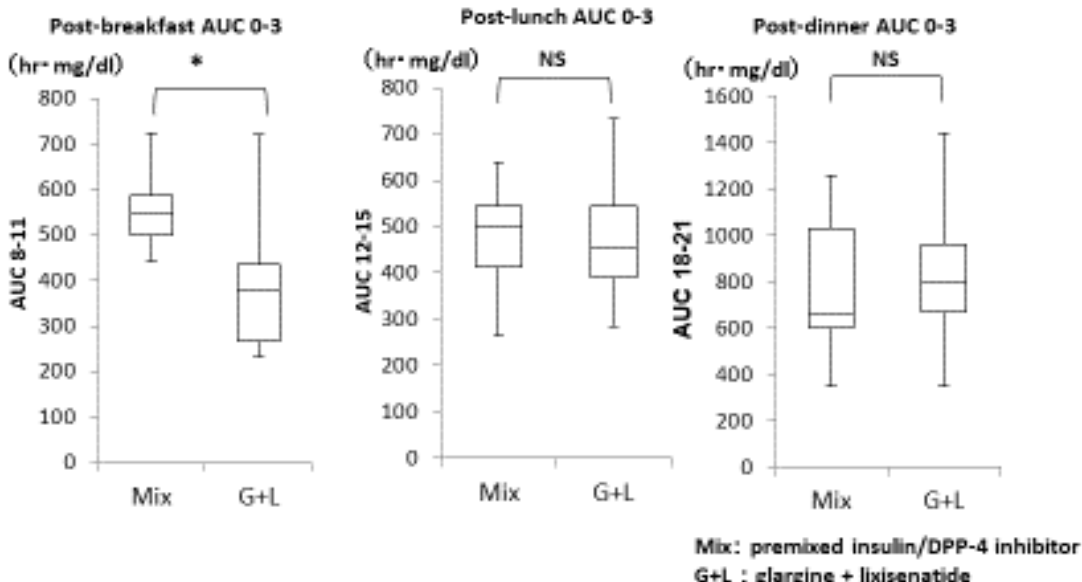

Figure 7. Comparison of twice-daily injections of premixed insulin/DPP-4 inhibitor and glargine/lixisenatide therapy for $0-3 \mathrm{~h}$ AUC after each meal (*p $<0.05$, NS: not significant)

Further, GA improved significantly in the study. It has already been reported that GA more accurately reflects postprandial hyperglycemia and the amplitude of glycemic excursions [10]. In our investigation, measured CGM data using actual test meals showed that the postbreakfast $0-3 \mathrm{~h}$ AUC improved significantly. This suggested that changing treatment by mainly focusing on postprandial glucose levels and, in particular, post-breakfast levels improves blood glucose. As $M$ values also improved significantly from 86.1 (41.0-104.5) to 37.2 (16.1-73.0), it appears that switching from twice-daily injections of premixed insulin with DPP-4 inhibitor to combined therapy with glargine and lixisenatide may lead to higher quality blood glucose control with smaller glycemic excursions. However, although HbAlc showed a tendency to improve, no significant changes occurred. In some cases, combined therapy with glargine and lixisenatide tended to offer insufficient control of blood glucose spikes compared to twicedaily injections of premixed insulin (Figure 7), and this may have been the reason for not achieving significant improvements in HbAlc levels.

Body weight decreased significantly as a result of switching treatment. Because the insulin dose did not change significantly, and no significant differences were noted between the two treatments for hypoglycemia, changing the insulin treatment regimen itself may not have affected the body weight. The body weight loss observed appeared to be mainly due to appetite suppression by GLP-1RA mediating the central nervous system and appetite suppression due to delayed gastric emptying. The present study evaluated and investigated detailed changes in dietary behavior accompanying switching treatment. Significant improvements were noted for dietary behavior due to switching treatment, with significant improvements noted for food motivation, eating as a diversion, and, in particular, diet regularity on the eating behavior questionnaire. The results from questions composing each section indicated significant improvements for parameters related to habits such as improvement in the amount of food bought and improvement of the habit of keeping food nearby. It appeared that the improvement of such dietary behavior led to decreased body weight. In addition, the trend for improvement noted in the hypoglycemia subscale of the DTR-QOL suggested that switching from basal insulin to lixisenatide reduced the tendency of overeating, including during dinner to prevent late-night hypoglycemia. This may have been advantageous to the decreases in body weight observed.

Investigation of the effects of treatment on daily lifestyle, which is a very important factor in the management for obesity and diabetes, indicated that switching treatment reduced anxiety regarding diabetes treatment and improved the level of satisfaction with treatment. This may have been the beneficial effect for both that body weight gain caused by twice-daily injections of premixed insulin with DPP4 inhibitor and unstable blood glucose fluctuation were improved by switching to combined therapy with glargine and lixisenatide. With regards to restrictions on social activities and daily lifestyle, which are important issues in insulin therapy, switching the method of treatment that two kind of injections (glargine and lixisenatide) only needed to be performed in the morning; this was a significant factor in improved QOL related to constraints on activities mainly involving going out and meal times. As a result, this appeared to contribute to improvement of how patients felt that self-injections of insulin had previously impaired their lifestyle.

However, although switching treatment led to a tendency for improvement in questions related to hypoglycemia, no significant differences were noted. Blood glucose control while receiving premixed insulin was insufficient so many patients had high fasting blood glucose levels and therefore were unlikely to fall into a hypoglycemic state. This may have been why no significant changes were noted for hypoglycemia as a result of switching treatment.

In our study, switching from twice-daily injections of premixed insulin with DPP-4 inhibitor to combined therapy with basal insulin and GLP-1 RA further improved blood glucose control, reduced body weight, and led to improvements in patient adherence and QOL including dietary behavior without any change in total insulin dosage $[11,12]$. These results could be highly significant for the future treatment of overweight patients with type 2 diabetes.

\section{Limitations}

This study had some limitations. As there was only a small sample of 10 patients and the treatment was switched over a short period of time, crossover research with a larger sample size and longer period of observation needs to be conducted in the future.

\section{Conclusions}

Obese patients with type 2 diabetes with insufficient blood glucose control from a regimen of twice-daily injections of premixed insulin to combined therapy with glargine and lixisenatide reduces glycemic excursions, improves blood glucose control, and may also inhibit weight gain by improving dietary behavior. Results also suggest that switching to this combined therapy can alleviate patient anxiety regarding treatment and anxiety in both regarding hypoglycemia in particular. 
Ochi F (2018) The comparative study with lixisenatide /glargine combination therapy versus twice-daily premixed insulin therapy/DPP-4 inhibitor on glycemic variability, and feeding behavior in obese patients with type 2 diabetes mellitus

\section{Disclosure}

None of the authors have any conflicts of interest associated with this research.

\section{References}

1. Inzucchi SE, Bergenstal RM, Buse JB, Diamant M, Ferrannini E, et al. (2012) Management of Hyperglycemia in Type 2 Diabetes, 2015: A Patient-Centered Approach: Update to a Position Statement of the American Diabetes Association and the European Association for the Study of Diabetes. Diabetes Care 38: 140-149.

2. Tokuda M, Ochi H, Miyakoshi K, Kubooka Y, Kusunoki Y, et al. (2013) Effects of Exenatide on the Glycemic Control and Eating Behavior in Obese Japanese Patients with Type 2 Diabetes Mellitus. J Japan Diab Soc 56: 759-768.

3. Meier JJ (2012) GLP-1 receptor agonists for individualized treatment of type 2 diabetes mellitus. Nat Rev Endocrinol 8: 728-742. [Crossref]

4. Seino Y, Min KW, Niemoeller E, Takami A; EFC10887 GETGOAL-L Asia Study Investigators (2012) Randomized, double-blind, placebo-controlled trial of the oncedaily GLP-1 receptor agonist lixisenatide in Asian patients with type 2 diabetes insufficiently controlled on basal insulin with or without a sulfonylurea (GetGoal-LAsia). Diabetes Obes Metab 14: 910-917. [Crossref]
5. Meier JJ, Nauck MA (2010) Is the diminished incretin effect in type 2 diabetes just an epi-phenomenon of impaired beta-cell function? Diabetes 59: 1117-1125. [Crossref]

6. Ishii H, Yamamoto T, OhashiY (2001) Development of insulin therapy related quality of life measure (ITR-QOL). J Japan Diab Soc 44: 9-15.

7. Ishii H (2012) Development and psychometric validation of the Diabetes TherapyRelated QOL (DTR-QOL) questionnaire. J Med Econ 15: 556-563. [Crossref]

8. Japan Society for the Study of Obesity (2006) Obesity Treatment Guidelines 2006. Journal of Japan Society for the Study of Obesity 12: 33-39.

9. Conrad Eng (2014): Lancet in the Sept p. 11.

10. Yoshiuchi K, Matsuhisa M, Katakami N, Nakatani Y, Sakamoto K, et al. (2008) Glycated albumin is a better indicator for glucose excursion than glycated hemoglobin in type 1 and type 2 diabetes. Endocr J 55: 503-507. [Crossref]

11. Iwamoto Y, Taniguchi T, Nonaka K, Okamoto T, Okuyama K, et al. (2010) Doseranging efficacy of sitagliptin, a dipeptidyl peptidase- 4 inhibitor, in Japanese patients with type 2 diabetes mellitus. Endocr J 57: 383-394. [Crossref]

12. Cornell S (2012) Differentiating among incretin therapies: a multiple-target approach to type 2 diabetes. J Clin Pharm Ther 37: 510-524. [Crossref]

Copyright: (C2018 Ochi F. This is an open-access article distributed under the terms of the Creative Commons Attribution License, which permits unrestricted use, distribution, and reproduction in any medium, provided the original author and source are credited. 